Revista Tecné, Episteme y Didaxis: TED. Año 2014, Número Extraordinario. ISSN Impreso: 0121-3814, ISSN web: 2323-0126

Memorias, Sexto Congreso Internacional sobre Formación de Profesores de Ciencias. 08 al 10 de octubre de 2014, Bogotá

\title{
Diseño teórico de investigación y propuesta de intervención para la enseñanza de la física, basada en los estilos enseñanza y estilos de aprendizaje de los estudiantes del grado $11 \mathrm{del}$ Colegio Guillermo Taborda, mediante la investigación dirigida
}

Ballesteros, Eudys', Martínez, Gina²Muñoz, Carmenza3, Villarreal, Jorge ${ }^{4}$

Categoría 2. Trabajo de investigación (concluido)

\section{Resumen}

El presente trabajo muestra una propuesta para el diseño y descripción de una estrategia didáctica para la enseñanza de la física en undécimo grado, basado en los estilos de aprendizaje de los estudiantes y teniendo en cuenta los estilos de enseñanza propuestos por Martínez; como resultado de una experiencia práctica e investigativa en el aula de clase de un plantel educativo de carácter privado en Medellín-Colombia; Para soportar esta investigación se implementaron los cuestionarios CHAEA y CEE, con el fin de identificar los diferentes estilos de aprendizaje que están inmersos en el aula y el estilo de enseñanza predominante en la enseñanza de la física. Los resultados dan cuenta de que existe una brecha entre los estilos de aprendizaje de los estudiantes y el estilo de enseñanza de la física.

\section{Palabras claves}

Estilos de Aprendizaje, Estilos de Enseñanza, Investigación Dirigida, Enseñanza de la Física, Formación de maestros.

\section{Presentación del problema}

En la experiencia práctica e investigativa que se realiza en un plantel educativo de carácter privado en la ciudad de Medellín-Colombia y de educación

\footnotetext{
1 Universidad de Antioquia. euballesteroscn@hotmail.com

2 Universidad de Antioquia. gimarce2103@hotmail.com

3 Universidad de Antioquia. carmen3189@gmail.com

4 Universidad de Antioquia. jorgevf2005@gmail.com
} 
Revista Tecné, Episteme y Didaxis: TED. Año 2014, Número Extraordinario. ISSN Impreso: 0121-3814, ISSN web: 2323-0126

Memorias, Sexto Congreso Internacional sobre Formación de Profesores de Ciencias. 08 al 10 de octubre de 2014, Bogotá

personalizada, que cuenta con una población de 197 estudiantes de estrato socioeconómico medio, se tomó como muestra el grado décimo conformado por 10 estudiantes en 2013 de edades entre los 15 y 17 años, se realizaron procesos de observación participante y no participante y se identificó que en clases magistrales sólo algunos estudiantes participaban activamente en las actividades propuestas, y que al implementar diferentes tipos de actividades que incluían la experimentación, la reflexión y la aplicación de ideas, aumentaba la participación.

A partir de esta observación se consideró que algo estaba pasando, que los estudiantes percibían las clases de diferente manera de acuerdo a la actividad que se planteara, es decir, que no todos aprendían de la misma manera.

Así, se realizó un rastreo bibliográfico para ver qué se encontraba acerca de las diferentes formas de aprender; y así fue como se encontraron algunos autores como Honey, Gallego y Catalina Alonso quienes vienen trabajando recientemente acerca de una temática importante para tener en cuenta dentro del aula de clase, llamada "estilos de aprendizaje", que sustenta que existen diversas formas de aprender, y aportan un cuestionario para su identificación llamado CHAEA, el cual fue aplicado a los estudiantes.

Los resultados de estas pruebas mostraron que entre los 10 estudiantes que conformaban el grupo están desarrollados los cuatro estilos de aprendizaje que describen los autores: Activo, reflexivo, teórico y pragmático.

De igual forma, se encuentra que es importante relacionar estos resultados con la forma de la enseñanza, por lo cual en la búsqueda de antecedentes se indaga sobre esta característica y se identifica que Martínez Geijo (2013) propone unos estilos de enseñanza y un instrumento de identificación de éstos, llamado CEE; el cual fue aplicado a la maestra titular del área de física de esta institución; para determinar si éste estaba acorde con los estilos de aprendizaje de los estudiantes. Se encontró que la docente tiene un estilo de enseñanza predominante formal, correspondiente al estilo de aprendizaje reflexivo. Concluyendo de esta manera, que la enseñanza de la física está enfocada sólo a uno de los cuatro estilos de aprendizaje que están presentes en el aula de clases, favoreciendo sólo a algunos de los 10 estudiantes.

Por tanto, se observa que hay una brecha entre los estilos de aprendizaje y los estilos de enseñanza; por lo cual en la búsqueda de mejorar la calidad educativa 
Revista Tecné, Episteme y Didaxis: TED. Año 2014, Número Extraordinario. ISSN Impreso: 0121-3814, ISSN web: 2323-0126

Memorias, Sexto Congreso Internacional sobre Formación de Profesores de Ciencias. 08 al 10 de octubre de 2014, Bogotá

de los estudiantes en el área de la física, desde sus propios estilos de aprendizaje, se parte del siguiente interrogante: ¿¿Qué características debe tener una estrategia didáctica de enseñanza para la física, basada en los estilos de aprendizaje de los estudiantes del grado 11 del Colegio Guillermo Taborda Restrepo?; con el objetivo de experimentar nuevos métodos de enseñanza que inciten al trabajo individual y grupal participativo, al igual que mostrar la relación que hay entre los estilos de aprendizaje y la metodología de la enseñanza, haciendo énfasis en la identificación de sus estilos de aprendizaje.

\section{Objetivos}

General: Caracterizar el diseño de una estrategia didáctica para la enseñanza de la física, basado en los estilos de aprendizaje de los estudiantes del grado $11 \mathrm{del}$ Colegio Guillermo Taborda Restrepo.

\section{Especificos:}

- Identificar los estilos de aprendizaje predominantes en los estudiantes, a partir del modelo seleccionado.

- Determinar las fundamentaciones teóricas que sustentan el problema y el diseño de la estrategia didáctica para la enseñanza de la física.

- Describir la metodología que se va a implementar en el diseño de la estrategia, teniendo en cuenta los estilos enseñanza y los estilos de aprendizaje de los estudiantes.

- Relacionar las características que hay en los estilos de aprendizaje de los estudiantes y el diseño de una estrategia didáctica para la enseñanza de la física basada en éstas.

\section{Marco de referencia conceptual}

Para poder llevar a cabo este trabajo, se ha elaborado un estado del arte y se han seleccionado algunas teorías que apoyan las propuestas aquí planteadas; razón por la cual, se citará en primera instancia el concepto "estilo de aprendizaje", visto desde la perspectiva de Honey y Alonso, en el 1994; y el concepto de "estilo de enseñanza" planteado por Martínez Geijo, en el 2002. Para posteriormente, dar cabida a otros elementos importantes para este trabajo.

Por tanto, para diversos autores los estilos de aprendizaje "Son los rasgos cognitivos, afectivos y fisiológicos que sirven como indicadores relativamente 
Revista Tecné, Episteme y Didaxis: TED. Año 2014, Número Extraordinario. ISSN Impreso: 0121-3814, ISSN web: 2323-0126

Memorias, Sexto Congreso Internacional sobre Formación de Profesores de Ciencias. 08 al 10 de octubre de 2014, Bogotá

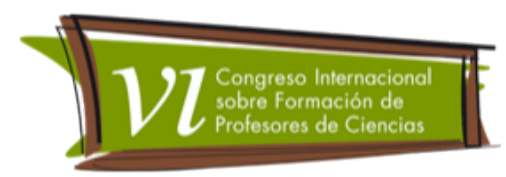

estables de cómo los alumnos perciben interacciones y responden a sus ambientes de aprendizaje"; son las distintas formas en que los estudiantes moldean los contenidos y conceptos, resuelven problemas de su entorno e interpretan la información (Gallego \& Nevot, 2008).

Los estilos de enseñanza, "Son las categorías de preferencias y comportamientos de enseñanza que el docente exhibe habitualmente en cada fase o momento de la actividad de enseñanza que se fundamentan en actitudes personales que le son inherentes, en que han sido abstraídos de su experiencia académica y profesional y en que tienen como referente los Estilos de Aprendizaje. Martínez (2002, 2007)" (Renes, Echeverry, Chiang , Rangel, \& Martinez Geijo, 2013, pág. 4)

Los estilos de enseñanza se conceptualizan con relación a los Estilos de Aprendizaje propuestos por Alonso, se pueden determinar y relacionar de la siguiente manera (Renes, Echeverry, Chiang, Rangel, \& Martinez Geijo, 2013).

Estilo de enseñanza abierto, favorecen al alumnado de estilo de aprendizaje activo. Los docentes se plantean nuevos contenidos sin ajustarse de manera estricta a algún tipo de planificación. En las clases hay actividades novedosas, problemas reales, trabajo en equipo, cambio de metodologías y de actividades, en fin, son creativos, improvisadores, innovadores, flexibles y espontáneos.

Estilo de enseñanza formal, favorecen al alumnado de estilo de aprendizaje reflexivo. Los docentes planifican de manera detallada el proceso de enseñanza por la cual se rigen de manera estricta. Elaboran la enseñanza con actividades detalladas, fomentan la reflexión y la sustentación racional en los estudiantes, se promueve el trabajo individual sin improvisación, son responsables, reflexivos, cuidadosos, tranquilos y con mucha paciencia.

Estilo de enseñanza estructurado, favorecen al alumnado con estilo de aprendizaje teórico. Se pone el énfasis en la estructuración de la planeación, con contenidos articulados en un marco teórico amplio, se evitan los cambios de metodología y gustan de solicitar demostraciones. Los docentes se caracterizan por ser objetivos, lógicos, perfeccionistas y sistemáticos.

Estilo de enseñanza funcional, favorece al alumnado de estilo de aprendizaje pragmático. Se pone el énfasis en la viabilidad de la planificación, mayor preponderancia de contenidos procedimentales, se emplean ejemplos en las explicaciones con poca clase magistral, partidarios del trabajo en equipo con instrucciones claras para evitar que se caiga en el error. Los docentes se 
Revista Tecné, Episteme y Didaxis: TED. Año 2014, Número Extraordinario. ISSN Impreso: 0121-3814, ISSN web: 2323-0126

Memorias, Sexto Congreso Internacional sobre Formación de Profesores de Ciencias. 08 al 10 de octubre de 2014, Bogotá

caracterizan por ser prácticos, realistas, concretos y con tendencia a rentabilizar su esfuerzo.

Para el diseño de las actividades en Física que tengan como base estos estilos, de aprendizaje y enseñanza, se toma como base metodológica de la enseñanza la investigación dirigida, ya que esta se considera una herramienta fundamental para el desarrollo de estos estilos. Esta metodología se define como "una estrategia para construcción del aprendizaje, además los estudiantes aplican metodologías que los llevan por caminos del auto aprendizaje y llegar así a la premisa de "aprender a aprender"' (Porlan \& Cañal, 1987), (Ruiz, 2012, pág. 24). Este método de investigación "se fundamenta en el desarrollo de una pregunta. Todo conocimiento o saber, inicia con una pregunta, pues esta, es la motivación que tiene un ser para profundizar un conocimiento determinado, del cual tiene algún manejo, pero es insuficiente cuando necesita explicar nuevas situaciones 0 problemas. Es importante, que las preguntas a desarrollar con los estudiantes, estén estrechamente relacionadas con sus necesidades e inquietudes de su entorno, pues esto garantiza el interés de avanzar en un saber específico". (Ruiz, 2012, pág. 24)

\section{Metodologia de trabajo en el aula}

Inicialmente se realiza la aplicación del test de Estilos de Aprendizaje a los estudiantes y el test de Estilos de Enseñanza a la docente. El análisis de los resultados de las aplicaciones llevo a la búsqueda de una estrategia que permitiera articular las características de lo encontrado con el proceso de enseñanza de los conceptos físicos.

Para llevar a cabo esta propuesta de intervención se tendrá en cuenta que la muestra es de 9 estudiantes, por tanto se organizará el grupo en tres subgrupos, los cuales serán conformados teniendo en cuenta el estilo de aprendizaje de cada uno, de tal manera que quede un estudiante por cada estilo.

Basándose en todo esto se pretende abordar el tema de hidrostática a partir de la Investigación Dirigida y en relación con el constructo:

1. Se presentara a los estudiante el experimento propuesto por Descartes llamado el Diablillo Cartesiano o Ludión, desde el cual se pueden trabajar conceptos como el Principio de Pascal, Principio de Arquímedes y Presión; 
Revista Tecné, Episteme y Didaxis: TED. Año 2014, Número Extraordinario. ISSN Impreso: 0121-3814, ISSN web: 2323-0126

Memorias, Sexto Congreso Internacional sobre Formación de Profesores de Ciencias. 08 al 10 de octubre de 2014, Bogotá

esto se llevará acabo inicialmente desde la construcción del instrumento y se orientara por medio de preguntas como:

¿En qué consiste el funcionamiento del ludión?

¿Qué aspectos físicos se evidencian en el comportamiento de Ludión?

2. Los estudiantes plantearan las observaciones realizadas sobre el experimento y formularan cuestionamientos a partir de la interacción con el instrumento.

3. Se llevara a cabo otro experimento con el fin de que establezcan relaciones a partir de estas dos experiencias.

4. Basándose en las experiencias se presentara un debate entre los tres equipos en donde cada uno expondrá las hipótesis generadas y las preguntas construidas, con el fin de determinar una teoría que se crea más asertiva al momento de explicar el fenómeno.

5. Luego del debate, cada equipo revisara, replanteara y complementara las ideas y aportes que se tenían para ir estructurando los conceptos relacionados con hidrostática.

6. Los estudiantes desarrollaran por fuera del aula una consulta e investigación que les permita soportar teóricamente las ideas construidas hasta el momento.

7. Se confrontaran las distintas investigaciones y cada grupo deberá pensar en una idea de actividad experimental diferente a las trabajadas en donde se haga uso de los conceptos hasta el momento construidos.

8. Después cada actividad experimental desarrollada por los grupos se distribuirá en grupos contrarios, para que estos piensen en posibles soluciones.

9. Cada grupo presentara los resultados sobre el proceso (toma de datos y observaciones, preguntas y cuestionamientos, planteamientos sobre las distintas situaciones experimentales y construcción de conceptos) con el fin de tener un registro sobre lo trabajado y construir un solo concepto sobre las temáticas relacionadas sobre hidrostática.

10. Se determinar dentro de los grupos de trabajo los tres tipos de evaluación: autoevaluación, Coevaluación y heteroevaluación, por medio de una rúbrica que permita dejar ver lo logrado en el proceso.

Como se puede ver esta metodología de enseñanza permite a los estudiantes por medio de la indagación, la experimentación, la creación y la reflexión, fortalecer la construcción de conocimiento y desarrollar habilidades indispensables para desenvolverse en la sociedad. 
Revista Tecné, Episteme y Didaxis: TED. Año 2014, Número Extraordinario. ISSN Impreso: 0121-3814, ISSN web: 2323-0126

Memorias, Sexto Congreso Internacional sobre Formación de Profesores de Ciencias. 08 al 10 de octubre de 2014, Bogotá

Debe quedar claro que en el proceso investigativo, teniendo en cuenta que se realiza como trabajo final de la licenciatura, se está en la fase del diseño de los instrumentos de recolección de información, por lo que la propuesta de diseño aún no se ha implementado, pero para poder construirla se parte de las características de los constructos base de la investigación, en este caso las teorías de Estilos de Aprendizaje y de Enseñanza.

\section{Metodología de investigación}

Teniendo en cuenta que lo que se pretende con esta investigación es hacer un diseño de una secuencia didáctica que tiene como base la investigación dirigida, y que este diseño se realiza en un grupo de 9 estudiantes, entonces el diseño se va aplicando en este caso y se busca describir las características del diseño relacionando la investigación dirigida con los estilos de aprendizaje y estos con el estilo de enseñanza que el docente debe utilizar. Por este sentido, en primer lugar utilizamos un proceso de razonamiento deductivo, ya que después de la identificación del problema de investigación se ha contrastado el esquema encontrado en el aula y compararlo con uno previo, es decir con un constructo teórico ya existente.

Así mismo, se puede afirmar que en este trabajo se parte de hipótesis sustentadas en el desarrollo teórico de la educación y la psicología, que son parte del esquema previo de comparación de la realidad del aula. A partir de esto podemos enmarcar nuestro trabajo en el enfoque empírico analítico de investigación, que tiene como características: el reconocimiento del conocimiento acumulado producto de la historia social, el partir de este conocimiento acumulado (estilos de enseñanza y aprendizaje), y el que se hagan uso de técnicas diversas (cuasi experimentos, observaciones, conjeturas racionales o tratamientos estadísticos).

Teniendo en cuenta los objetivos de esta investigación, este estudio es descriptivo, ya que tiene como finalidad decir cómo es y cómo se manifiestan diversos fenómenos (estilos de aprendizaje y enseñanza) en los grupos o personas que fueron objeto de la observación y la intervención.

Según el tratamiento que se ha hecho de la variable tiempo, el estudio que se presentan tienen un diseño transversal, ya que se recoge la información a analizar en un solo momento, un diseño, y no se realiza un seguimiento temporal de los fenómenos. 
Revista Tecné, Episteme y Didaxis: TED. Año 2014, Número Extraordinario. ISSN Impreso: 0121-3814, ISSN web: 2323-0126

Memorias, Sexto Congreso Internacional sobre Formación de Profesores de Ciencias. 08 al 10 de octubre de 2014, Bogotá

Conclusiones preliminares

1. Se logran determinaciones entre los enlaces de estilos y la investigación dirigida que se ha aplicado, en tanto se observa un avance en el trabajo de aula.

2. Se representa mediante la evaluación un avance en aportes teóricos que han sido relevantes en el momento de entender el trabajo individual y su vínculo con cada uno de los estilos de aprendizaje.

3. Por medio de las actividades damos sentido al concepto de experiencia y experimentación, en tanto buscamos hacer un contraste entre lo vivenciado y los planteamientos teóricos.

\section{Referencias bibliográficas}

Alonso, C. M. (1992). Estilos de aprendizaje: análisis y diagnóstico en estudiantes universitarios. Universidad Complutense.

Campanario, J. M., \& Moya, A. (1999). ¿Cómo enseñar ciencias? Principales tendencias y propuestas. Enseñanza de las Ciencias, pág. 179-192.

Cazau, P. (2004). Estilos de aprendizaje: Generalidades. Clidet .

Escurra, L. M. (2011). Análisis psicométrico del Cuestionario de Honey y Alonso de Estilos de Aprendizaje ( Chaea ) con los modelos de la Teoría Clásica de los Tests y de Rasch. Universidad de Lima, pág. 71-109.

Gallego, D. J., \& Quintanal, F. (2011). Incidencia de los estilos de aprendizaje en el rendimiento académico de la física y química de secundaria. Revista Estilos de Aprendizaje, pág. 198.

Gallego, D., \& Nevot, A. (2008). Los estilos de aprendizaje y la enseñanza de las matemáticas. Revista Complutense de Educación, 19 (1), pág. 95-112.

Geijo, P. M., \& Echeverry, L. M. (2013). Estilos de enseñanza: Un paso adelante en su conceptualización y diagnóstico.

Gil, D. J., \& Luna, A. N. (2007). Los estilos de aprendizaje y la enseñanza de las matematicas.

Muñoz, O., Rodríguez, R., \& Plaza, R. (2003). Análisis de los estilos de aprendizaje predominantes entre los estudiantes de ciencias de la salud. Enfermería Global , pág. 1-6. 
Revista Tecné, Episteme y Didaxis: TED. Año 2014, Número

Extraordinario. ISSN Impreso: 0121-3814, ISSN web: 2323-0126

Memorias, Sexto Congreso Internacional sobre Formación de Profesores de Ciencias. 08 al 10 de octubre de 2014, Bogotá

Nevot, A. (2004). Enseñanza de las Matemáticas basada en los estilos de aprendizaje. Bol. Soc. Esp. Mat. Apl n²8, pág. 119-184.

Porlan, R., \& Cañal, P. (1987). Investigando la realidad proxima: un modelo didáctico alternativo. Enseñanza de las ciencias, pág. 89-96.

Renes, P., Echeverry, L., Chiang, M. T., Rangel, L., \& Martinez Geijo, P. (2013). Estilos de enseñanza: Un paso a su conceptualización y diagnóstico. Revista Estilos de Aprendizaje, 11 (1), pág. 1-18.

Rodríguez, J. (2006). Validación del Chaea en estudiantes universitarios. Memorias , pág. 121-138.

Ruiz, L. A. (2012). La investigacion dirigida como estrategia metodológica, para orientar prácticas experimentales de Bíologia, el la básica secundaria, de la Sede Educativa Bachillerato Patía del Municipioi del Patía- Cauca. Universidad Nacional de Colombia, pág. 1-63. 